entirely correct, it would indicate that a surface concentration of thorium of approximately half the present amount might make it a little easier for the tube to operate with currents of the order of $10^{-12}$ amp. With a current of $6 \times 10^{-12}$ amp. and the corresponding $d V / d t$ of 0.3 volt per sec., operation was very reliable.

The upper limit of counts per minute was set mainly by the mechanical properties of the particular message register used. Increasing the capacity $C_{t}$ decreased the counting rate for a given photoelectric current the amount expected from the equations and at the same time it increased the minimum current required for operation nearly in proportion. One would conclude from this that as long as the capacity $C_{t}$ is less than about 200 micromicrofarads the period $T$ will not exceed twenty to thirty minutes if the tube is to operate at all. Attention should perhaps be called to the fact that the average counts as shown in Fig. 4 were all based on thirty or more counts for each observation.

\section{Remarks on Operating Characteristics}

Over the range of plate current from 1.0 to 15 mils, the tube drop was observed to remain nearly constant at 100 volts while an increase of current to 20 mils resulted in an increase in tube drop. An observable increase in tube drop took place in a few minutes time when the tube was carrying 15 mils which seems to indicate that 10 mils is probably a safe maximum average current. At- tention should be called to Table I for here a maximum current of only 4 mils is recommended by the manufacturer.

Although WL-759 tubes can be started with as low as 220 volts on the principle anode $P$, the current required by the starting electrode is large. The minimum plate voltage for good operation is 250 volts and the maximum about 325 volts.

These tubes were operated as "relaxation" oscillators up to a frequency of 300 operations per second which indicates that the maximum counting rate which can be used in a circuit similar to that of Fig. 1 will be set by the properties of the message register. Of course, for high speed operation due consideration must be given the selection of the resistance $R_{p}$ and the capacity $C_{p}$.

In every installation using WL-759 tubes under control of currents of the order of $10^{-11}$ amp., the greatest of care must be exercised in order to eliminate stray leakage currents which are likely to flow through mechanical supports of the tube, photo-tube or other auxiliary apparatus. Polarization effects, stray light and stray electrical disturbances must be reduced to a minimum by the proper selection of materials and construction of shielding.

I wish to thank Dr. H. C. Rentschler of the Westinghouse Electric and Manufacturing Company for introducing me to these very interesting "trigger" tubes and hope that future users will derive help from these studies.

\title{
The Design of a Magnetic Beta-Ray Spectrometer
}

\author{
J. L. LAWSON AND A. W. Tyler \\ University of Michigan, Ann Arbor, Michigan
}

(Received July 7, 1939)

\begin{abstract}
A magnetic beta-ray spectrometer of high resolution has been constructed. The theory of the resolution of this instrument has been investigated in detail and applied to the design of the slit system. The effects of scattering within the vacuum chamber have been studied and reduced to a negligible value. The corrections for the efficiency of the Geiger-Miiller counter and for the absorption in the counter window have been obtained as a function of the momentum of the incoming particles.
\end{abstract}

\section{INTRODUCTION}

$\mathrm{O}$ $\mathrm{NE}$ of the most accurate methods of measuring the momentum of a charged particle is by means of its deflection in a magnetic field.
This may be accomplished most accurately by means of a magnetic spectrometer. The path of a charged particle moving in a plane perpendicular to a uniform magnetic field is a circle. The 
product of the magnetic field intensity and the radius of the circular path is directly proportional to the momentum of the particle. Thus various momenta may be selected by changing either the radius of the selected paths or the value of the magnetic field. The first method is used in a photographic spectrometer. The system of baffles used in this case is unique in that a large momentum range can be investigated at one time. All particles within this momentum range are approximately focused on the photographic plate in positions corresponding to their momenta. The variable field spectrometer has a narrow exit slit in place of the photographic plate. Thus particles passing through this exit slit have a momentum proportional to the magnetic field intensity. This type of spectrometer can be made to have a better resolution for a given intensity than the former. Furthermore a Geiger-Müller counter or an ionization chamber may be used as a detector. These detectors are much more satisfactory than a photographic plate for measuring the spectra of natural radioactive materials since they will detect and measure a smaller quantity of radiation. The corrections which must be made for the variation in sensitivity of the detector with the energy of the particles may be determined more accurately for the Geiger-Müller counter than for either of the other detectors.

When the variable field spectrometer is constructed with the source and detector entirely within the region of uniform magnetic field, approximate focusing takes place after the particles have traversed a semicircle. N. F. Barber ${ }^{1}$ has shown that approximate focusing may be obtained with less than a semicircular path if the source and detector are moved outside of the magnetic field. This construction has the advantage that the detector can be shielded more completely from the direct radiation from the source because of their greater separation. However it is very difficult to make absolute measurements with this instrument because of the effect of the fringing flux. The variable field spectrometer of the $180^{\circ}$-focusing type was chosen as the most practical instrument. If the average radius of curvature of the particles is large, several inches of lead may be placed be-

${ }^{1}$ N. F. Barber, Proc. Leeds Phil. Soc. 2, 427 (1933). tween the source and detector to reduce the direct radiation to a negligible value.

\section{The Magnetic Field}

Much of the success of a magnetic spectrometer depends upon the production of a suitable magnetic field. The usual practice has been to use an iron core magnet, either permanently magnetized, or magnetized by means of a constant current flowing in an exciting coil. A permanent magnet can be relied upon to maintain a constant field over long periods of time, without a complicated mechanism to stabilize its exciting current. However, its field cannot be changed to a desired value as easily as can the field of an electromagnet. Therefore a permanent magnet is well suited to a photographic spectrometer, whereas an electromagnet is to be preferred for a spectrometer in which the radius is kept constant and the field varied. The initial cost of an electromagnet is considerably smaller than that of a permanent magnet of similar dimensions. The only real disadvantage is the need for providing an accurate control for the exciting current. Since this can be accomplished by means of a proper vacuum tube circuit, a magnet of this type has been selected as the one most suitable for the intended purpose.

The core is built from a good grade of soft iron with an approximate cross section of 200 square inches. The poles are 16 inches in diameter, tapering in the last inch to a 15 -inch diameter. Exciting coils are wound from $1 \times 0.01$ inch copper strap. Four such coils, 3 inches deep, are mounted on each pole. Provision has been made to include water cooling pipes if necessary. The total weight is approximately two tons.

The gap between poles is about $2 \frac{9}{16}$ inches and can be altered, if desired, by shims. This allows room to slide in a chamber of over-all dimensions up to $2 \frac{1}{2}$ inches. With this pole gap 80 watts of power yields a magnetic field of 1000 gauss, which is ample for the measurement of most spectra. The magnet has been shimmed until the field is constant to better than one part in 3000 on a radius of $5^{\prime \prime}$. Fig. 1 shows the dependence of the vertical magnetic field upon the distance from the center of the pole face. It can 


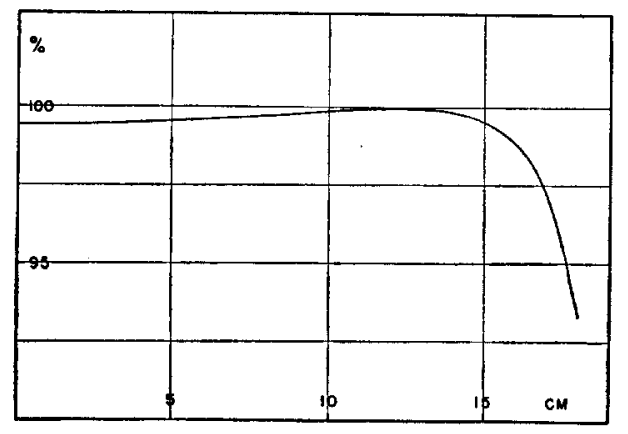

FIG. 1. Radial variation of the magnetic field intensity.

be seen that the field intensity reaches a very flat maximum at a radius of about $12 \mathrm{~cm}$. Consequently this value was chosen as the approximate radius of curvature of electron paths to be selected by the spectrometer.

The problem of magnet excitation is one requiring a considerable amount of care. Storage batteries can be used to supply the necessary power, but the current delivered by such batteries continually decays. It is not only desirable but almost essential to provide some means of quickly adjusting the current close to some specified value. Furthermore this current must remain within very close limits for any time interval. The limits on this variation depend upon the type of spectrum being measured, and in general should be no larger than one part in 1000. A current control device which satisfies all of these requirements has been constructed. A detailed description of this stabilizer has been published.*

A convenient and absolute method of measuring the magnetic field intensity is by means of a "flip coil" and ballistic galvanometer. The electric charge developed when the coil cuts a known area of magnetic flux is measured with a ballistic galvanometer. The charge sensitivity of the latter can be obtained by means of a standard mutual inductance. Five individual measurements are therefore necessary for an absolute determination of the magnetic field : the mutual inductance and its primary current, the turns $\mathrm{cm}^{2}$ of the flip coil, and two galvanometer deflections. The mutual inductance has been calibrated by the National Bureau of Standards and specified to be 10.79 millihenries with an

\footnotetext{
* J, L. Lawson and A. W. Tyler, Rev. Sci. Inst. 10, 304
(1939).
}

absolute accuracy of 1 part in 1000 . The primary current is measured with a potentiometer and standard resistance hence its contribution to the error is negligible. Two flip coils were made, each having a single layer of copper wire wound on a brass form. They were checked against one another several times and found to give consistent results within the assigned error in the turn $\mathrm{cm}^{2}$ of each, which was about 1 part in 3000. The galvanometer deflections can be read to about 1 part in 1500 on the average. Thus an absolute measurement of the magnetic field intensity can be made to about 1 part in 500 . Relative fields can be compared to better than 1 part in 1000 .

\section{The Geiger-Müller Counters}

The Geiger-Müller counters should be constructed so that a correction can be made for the variation in sensitivity of the counter with the energy of the incoming particles. If the beam of particles is made to pass completely through the cathode the sensitivity of the counter can be computed from the specific ionization of the particles. The cathode should be sufficiently large to encompass the beam and sufficiently long so that particles have a high probability of forming ions within the counting volume.

After a Geiger-Müller tube is assembled, it generally requires some treatment in order to perform satisfactorily. A number of processing methods have been described, but a procedure similar to that given by Duffendack, Lifschutz and Slawsky ${ }^{2}$ has been found to be most satisfactory in yielding good counters. Recently a simplified processing technique has been adopted. The counter is first cleaned with nitric acid, washed very thoroughly with distilled water ( 4 or 5 washings may be necessary), and dried with acetone: It is then sealed on a vacuum system, and sputtered in hydrogen for an hour or longer. With careful cleaning, the sputtering has not been found necessary, but is always helpful in obtaining clean elements.

A counter whose elements have been cleaned in this fashion has been used over a period of three months with no noticeable change in characteristics. Its cathode was a cylinder $18 \mathrm{~mm}$

\footnotetext{
2 Duffendack, Lifschutz and Slawsky, Phys. Rev. 52, 1231 (1937).
} 
long and $18 \mathrm{~mm}$ in diameter wound up from No. 18 copper wire. The anode was made from $0.003^{\prime \prime}$ tungsten wire, spot welded to a heavier tungsten lead. These elements were supported in a glass envelope, which was fastened with Picein wax to a brass plate containing a thin window. Internal glass shields were placed around the tungsten leads to reduce leakage between electrodes. The counter has been opened to the atmosphere several times during this three-month interval and no subsequent cleaning has been found necessary. Moreover this counter has been assembled with rubber hose vacuum connections.

Various gases have been used in GeigerMüller counters. Of all the gases tested in this laboratory, hydrogen has been found the most satisfactory. In the counter just described commercial hydrogen has been used at pressures up to $25 \mathrm{~cm}$ of mercury. A small amount of air mixed with the hydrogen does not impair the operation of the counter.

Since it is possible to make counters that can be treated in this fashion, the problem of obtaining a thin window to permit the entry of beta-particles is greatly simplified. The scattering and absorption of low energy particles by the gas between the source and detector can be very serious, and should be reduced to as small a value as possible. This is accomplished by placing the source in an evacuated tank, so that the particles travel almost the entire distance to the detector in a vacuum. A window must be placed over the exit slit to admit the particles to the gas of the counter. In order to reduce the scattering and absorption by the window, it should be made as thin as possible with a material of low atomic number. Moreover, it should be placed close to the counting volume, so that even if particles are scattered by the window, a large fraction of them will be recorded. If the Geiger-Müller tube is pumped down simultaneously with the vacuum tank, and then filled with hydrogen gas, the maximum pressure which the thin window must stand is the pressure of the hydrogen gas in the counter. This permits the use of exceedingly thin windows. Some workers have supported very thin windows on grills, enabling thinner windows to be used with the same pressure differential. This technique is questionable, however, for it introduces scattering for which corrections are very difficult to make. On the other hand, scattering and absorption by the window itself can be measured and the appropriate corrections computed.

The most satisfactory windows which have been tried are made from thin films of pyroxylin. They are very strong, easily made and their thickness can readily be measured by interference methods. The pyroxylin is carefully cleaned, then dissolved in dry amyl acetate. A small quantity of the solution is run onto a water surface. The solution spreads quickly and in a few seconds dries into a thin film. Before the film is quite dry it may be stretched to cover a large area. A wire frame, previously immersed in the water, is then pulled slowly out of the water vertically so that the film falls down on both sides forming a double sheet. The thickness of the film can be regulated by the amount of solution used. Ordinarily the total thickness of the double film cannot be made more than onehalf micron which is too thin to hold much pressure. The frame can be reimmersed in the water carrying its double film and another double layer added in the same manner. This process can be carried on almost indefinitely. The thin film is waxed between two plates through which the exit slit is milled. The whole assembly then can be waxed to the glass envelope of the counter. In this fashion, pyroxylin films as thin as $1 \frac{1}{2}$ microns have been made to withstand a pressure of $30 \mathrm{~cm}$ of mercury over a rectangle $4 \mathrm{~mm}$ by $16 \mathrm{~mm}$. One such film has been used steadily for three months and has never been replaced. During this period the vacuum tank has been opened to the atmosphere many times.

The performance of a Geiger-Müller counter is greatly affected by the electrical circuit in which it is located. If the potential is supplied to the counter through a large quenching resistor, the charging currents to the tube are quite low and the cycle of events occurring in a discharge takes place slowly. This causes serious losses in recording particles arriving in the counter at even moderate rates.

The Neher-Harper circuit ${ }^{3}$ has become widely

${ }^{3}$ H. V. Neher and W. W. Harper, Phys. Rev. 49, 940 (1936). 
known for the speed with which the counter discharges are extinguished. In this circuit the Geiger-Müller tube current changes the grid bias on a vacuum tube. The resulting change in plate current lowers the potential on the counter to its threshold value, thereby extinguishing the discharge. Due to the amplification of the vacuum tube, the charging currents to the tube capacities are high, resulting in a rapid extinction of the discharge.

Certain other circuits have been suggested for the control of Geiger-Müller tubes. The NeherPickering ${ }^{4}$ modification has certain commendable points, but unfortunately contains no provision for varying the grid bias on the extinguishing tube. The desirability for this variation has been pointed out and a suggestion for the inclusion of this adjustment has been made. ${ }^{5} \mathrm{~A}$ multivibrator extinguishing circuit has also been developed, ${ }^{6}$ but unfortunately tests have shown these circuits to be very critical in adjustment, and generally less satisfactory than the NeherHarper circuit.

Certain tests $^{7}$ with the conventional NeherHarper circuit show that the counting characteristics are affected by nearby charges, unless the counter is placed in a shield at approximately the same potential as the anode. It is usually convenient to make this shield at ground potential, which can be accomplished by "inverting" the circuit, or grounding the positive terminal of the high voltage supply. Such a circuit has proven very satisfactory.

Two general methods have been used to record the number of particles initiating the discharges in the Geiger-Müller tube. The voltage pulses produced in the counter can be recorded on a mechanical tabulator, or the average rate at which these pulses occur may be read on a frequency meter. ${ }^{8}$ Each method for recording counts has advantages which adapt it for certain uses. Where the counting rate is high, the frequency meter is the simplest and easiest system to use. However it is impossible to use a frequency meter for registering extremely small

\footnotetext{
${ }^{4}$ H. V. Neher and W. Pickering, Phys. Rev. 53, 316 (1938).

A. W. Coven, Rev. Sci. Inst. 10, 43 (1939).

I. A. Getting, Phys. Rev. 53, 103 (1938).

${ }^{7} \mathrm{~J}$. L. Lawson and A. W. Tyler, Phys. Rev. 53, 605 (1938).

${ }^{8}$ F. V. Hunt, Rev, Sci. Inst. 6, 43 (1935).
}

counting rates, therefore it is necessary to adopt the former method for beta-ray spectrometer applications. In order that the mechanical recorder is not subject to excessive counting rates, the pulses are scaled down by a scale-ofsixteen vacuum tube circuit, which has been previously described. ${ }^{9}$

With a constant ionizing source the recorded counting rate is a function of the potential applied to a Geiger-Müller counter. In order to realize constant counter sensitivity, therefore, it is necessary to apply a constant potential to the tube. This has been done by a stabilizer operating on the same principle as the magnet current stabilizer. A fraction of the output potential is balanced against a small battery. The residual voltage is amplified and used to modulate the grid of a vacuum tube, which acts as a variable series resistor in the high voltage lead. The effective series resistance of this tube is automatically varied so that it maintains a nearly constant output voltage. With this stabilizer potentials up to 2000 volts can be maintained over long lengths of time within an accuracy of one part in 1000 .

There are inevitable losses in the number of pulses which are recorded due to the rate at which they occur. For very fast counting rates, these losses may be so large that measurements are almost meaningless unless accurate corrections are known. These losses have been reduced by introducing a large amplification between the Geiger-Müller counter and the recorder, as suggested by Lifschutz and Duffendack. ${ }^{10}$

\section{The Vacuum Tank and Slit System}

A vacuum tank has been constructed which is $9^{\prime \prime}$ wide, $16^{\prime \prime}$. long, and $2 \frac{1}{2}{ }^{\prime \prime}$ deep over-all. The side walls are brass, and the top and bottom covers are $\frac{1}{2}^{\prime \prime}$ aluminum plate. Thus the inside vertical clearance is $1^{\prime \prime}{ }^{\prime \prime}$. The covers, as well as all attachments, are sealed to the side walls with rubber gaskets. A drawing of the finally adopted assembly is shown in Fig. 2.

The vacuum tank must contain a system of baffles to define the momentum of the selected

\footnotetext{
${ }^{9}$ H. Lifschutz and J. L. Lawson, Rev, Sci. Inst. 9, 83 (1938).

${ }_{10} \mathrm{H}$. Lifschutz and O. S. Duffendack, Phys. Rev. 54, 714 (1938).
} 


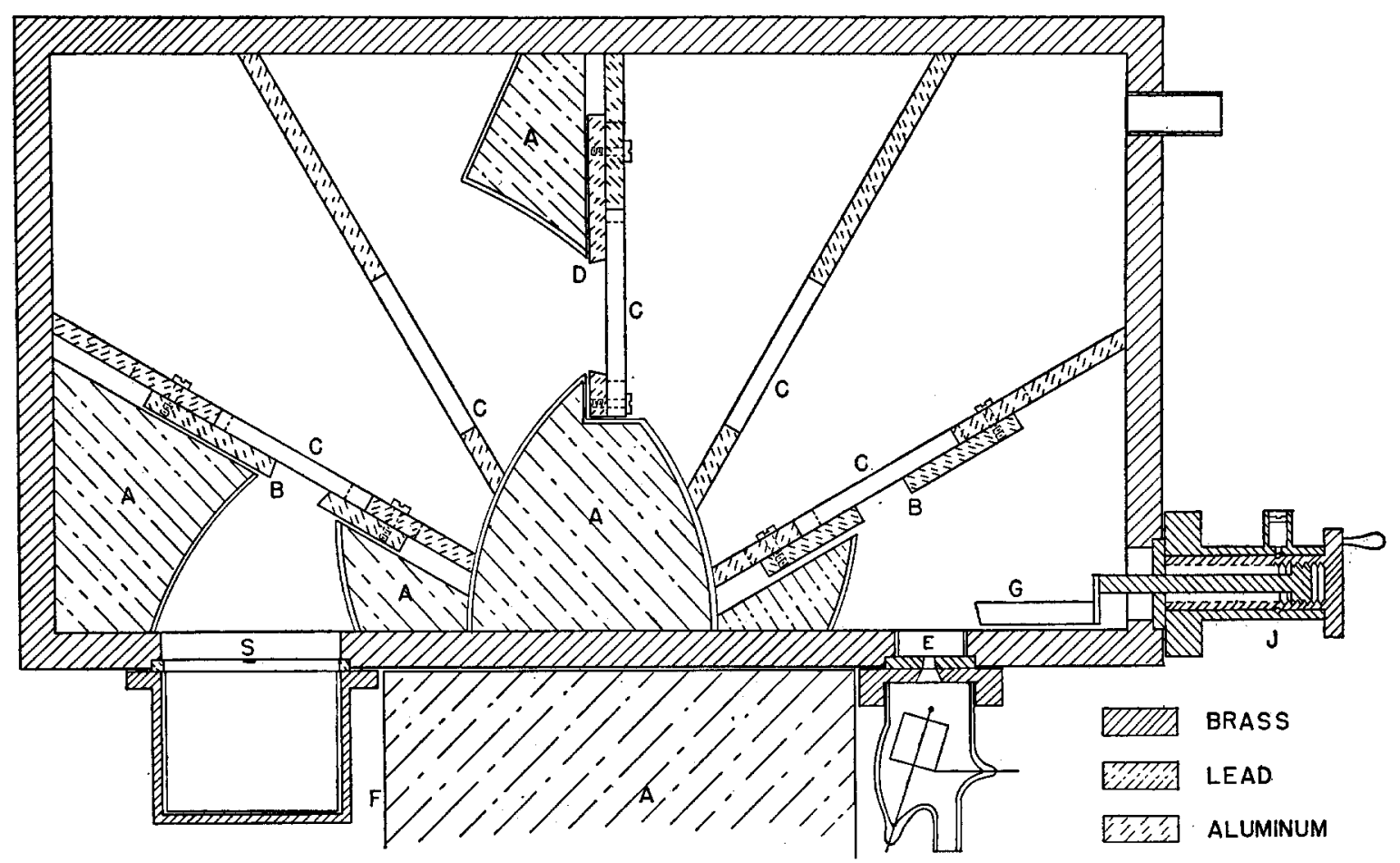

Frg. 2. The vacuum tank assembly.

particles. In addition to the central defining slit, $D$, two other slits, $B$, are installed in order to limit the number of particles which may be scattered into the exit slit, $E$, from various sections of the tank. The slits, $C$, which define the height of beam, serve to reduce scattering from the aluminum top and bottom of the chamber. Aluminum covered lead blocks, $A$, are liberally used to absorb gamma-radiation, but are always placed in such positions that particles cannot enter the exit slit after single scattering from these pieces. The entire inside of the tank is lined with aluminum or Bakelite to reduce the effects of scattering from the walls. Near the exit slit, $E$, a movable gate, $G$, is installed to cut off the beam, in order to determine the background counting rate. This gate can be controlled externally due to the vacuum grease joint, $J$.

Scattering from the edges of the one-inch hole surrounding the exit slit was easily tested by observing the manner in which the beam was cut off as the gate, $G$, was moved across the opening. There was noticeable scattering when this hole was lined with lead, but negligible scattering when the lining was made of Bakelite. $165-\mathrm{kv}$ beta-particles from radioactive phosphorus were used for this test.

Originally the source was mounted in a similar hole in the tank wall, but the scattering from the edges of this hole could not be reduced sufficiently by lining it with Bakelite. A typical test with $165-\mathrm{kv}$ particles showed that, when a brass block in front of the source obscured the direct beam, $3.9 \pm 0.3$ percent of the original number of particles were still recorded. Tests were carried out to show that this was due to scattering from the edges of the one-inch hole. This indicates how serious the scattering from a defining slit would be if it were placed close to the source. Consequently the rectangular hole shown in the diagram was milled from the tank wall. The source, $S$, is mounted on an aluminum frame which fits into a corresponding slot in the tank wall. A box, $F$, lined with Bakelite, is bolted over the whole assembly. The entire tank and slit system is so arranged that no singly scattered particles can enter the exit slit except those scattered from the back wall of the box, $F$, and the edges of the defining slits. With this arrangement, a brass block in front of the source ob- 
scuring the direct beam reduced the recorded number of $165 \mathrm{kv}$ particles to $0.11 \pm 0.2$ percent of $i$ ts original value.

The source in a photographic spectrometer is usually tilted with respect to the plane of the photographic plate, in order to secure a better line shape. This procedure has often been carried over to the variable field spectrometer. Essentially the same improvement in line shape can be realized without tilting and without loss of intensity, by making the source narrower and correspondingly thicker. Under these conditions the effective thickness of either source is the same. The effective thickness of the source should be made as small as possible, in order to minimize absorption and scattering. It is easier to prepare and mount an effectively thin source in the plane of the exit slit.

The scattering due to gas in the chamber can be easily detected. In a typical test, the introduction of air to a pressure of $2.7 \mathrm{~cm}$ of mercury reduced the recorded intensity of $165 \mathrm{kv}$ particles to less than half of its original value. Since scattering is much more pronounced for lower energy particles, the measurement of the very low energy regions of a spectrum requires the use of a well-evacuated tank.

\section{Theory of Resolution}

The magnetic field and slit system just described selects particles within a definite momentum range. If the slits are adjusted so that this range is fairly large, which is sometimes necessary to secure a measurable intensity, some method of averaging must be employed to determine the specific momentum which the measurement represents. The total number of particles involved in each measurement depends on the distribution in momentum of particles leaving the source and on the effective solid angle subtended by the exit slit. This solid angle, which will hereafter be called the transmission factor of the slit system, is a function of momentum and must be calculated in order to average the momentum.

This problem was treated originally by W. A. Wooster ${ }^{11}$ and more recently by $\mathrm{K}$. T. Li., ${ }^{12}$ They have solved the problem for the case of the

\footnotetext{
${ }^{11}$ W. A. Wooster, Proc. Roy. Soc. A114, 729 (1927).
}

${ }_{12}$ K. T. Li, Proc. Camb. Phil. Soc. 33, 164 (1937). slit system generally used with a photographic spectrometer. Their results may also be used to obtain an approximate transmission factor for the variable field spectrometer. However, it was considered worth while to derive the formulae directly from the geometrical arrangement of the source and slits used in this type of spectrometer in order to determine the optimum relative sizes of the source and slits as well as a more accurate transmission factor.

The essential elements of the system used in the present instrument are as follows: In the coordinate system $x y z$ a rectangular source of width $l_{0}$ is mounted in the $x y$ plane with its center at the origin. A rectangular exit slit of width $l$ is located in the $x y$ plane with its center at $x=2 p$. A rectangular defining slit of width $D$ is located in the plane $x=p$ with its center at the point $(p, o, p)$. The source and slits are all of the same height, $h$. A uniform magnetic field, $H$, is applied in the $y$ direction. (Fig. 3.)

The equations of motion of a particle leaving the source are readily obtained as:

$$
\begin{gathered}
{\left[\dot{z}_{0}-\omega\left(x-x_{0}\right)\right]^{2}+\left[\dot{x}_{0}+\omega\left(z-z_{0}\right)\right]^{2}=\dot{x}_{0}{ }^{2}+\dot{z}_{0}{ }^{2},} \\
y=\dot{y}_{0} t+y_{0},
\end{gathered}
$$

where $x_{0} y_{0} z_{0}$ are the coordinates and $\dot{x}_{0} \dot{y}_{0} \dot{z}_{0}$ are the velocity components of the particle as it leaves the source and $\omega=e H / m c$. The intersection of the path with the $x y$ plane is found by setting $z=0$ in Eq. (1).

$$
x-x_{0}=2 \frac{\dot{z}_{0}}{\omega},
$$

since $z_{0}$ is always zero. Eqs. (2) and (3) give the coordinates of the particle as it passes through

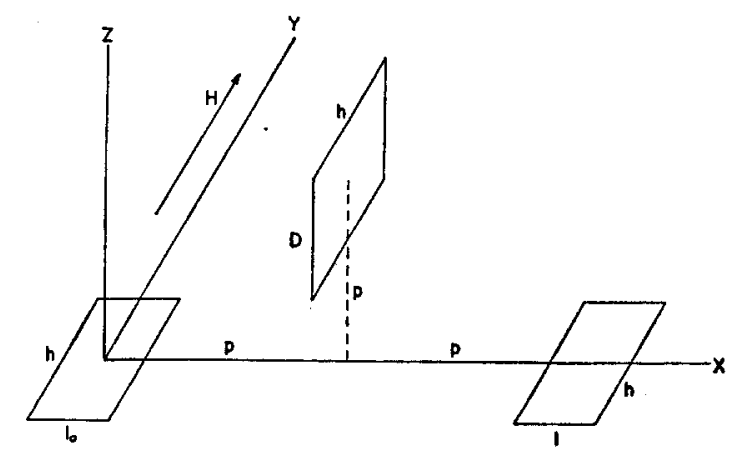

FIG. 3. The fundamental slit system. 
the plane of the exit slit in terms of the original position and velocity components.

The expression for the intensity may be obtained from them more easily by introducing the angle coordinates, $\phi, \theta, \psi$ and $\alpha$ defined as follows :

$$
\begin{aligned}
\cos \theta & =\dot{z}_{0} / v, \\
\sin \phi \sin \theta & =\dot{y}_{0} / v=\sin \alpha, \\
\cos \theta & =\cos \alpha \cos \phi .
\end{aligned}
$$

$\theta$ and $\phi$ are the usual angular spherical coordinates, $\psi$ is the projection of $\theta$ on the $x z$ plane, and $\alpha$ is the angle of inclination of the path with respect to the $x z$ plane. $\psi$ and $\alpha$ form the two sides and $\theta$ the hypotenuse of a right-angled spherical triangle of which $\phi$ is one angle. In these coordinates Eqs. (2) and (3) become:

$$
\begin{gathered}
x-x_{0}=2 \frac{v}{\omega} \cos \theta, \\
y-y_{0}=v t \sin \alpha=\frac{v}{\omega}(\pi-2 \psi) \sin \alpha .
\end{gathered}
$$

The number, $n_{v}$, of particles of velocity, $v$, per unit solid angle per square $\mathrm{cm}$ per second, leaving a thin source is essentially constant. The total number per second leaving the source is

$$
N=\iiint \int n_{v} \sin \theta d \theta d \phi d y_{0} d x_{0}
$$

or in terms of $\theta$ and $\alpha$

$N=\iiint \int n_{v} \frac{\cos \alpha \sin \theta}{\left(\sin ^{2} \theta-\sin ^{2} \alpha\right)^{\frac{1}{3}}} d \theta d \alpha d y_{0} d x_{0}$.

This equation may be integrated with respect to $\alpha$. It is essentially an integration in $y$ over the height of the exit slit. The limits in $\alpha$ can be obtained from Eq. (8). The result is simplified considerably if $\pi-2 \psi$ in Eq. (8) is replaced by $\pi-2 \theta$. It can be shown that this approximation has a negligible effect on the results. The result of the first integration is:

$$
\begin{array}{r}
N=\iiint n_{v} \sin \theta\left[\sin ^{-1} \frac{(h / 2)-y}{A}\right. \\
\left.+\sin ^{-1} \frac{(h / 2)+y}{A}\right] d \theta d y_{0} d x_{0}
\end{array}
$$

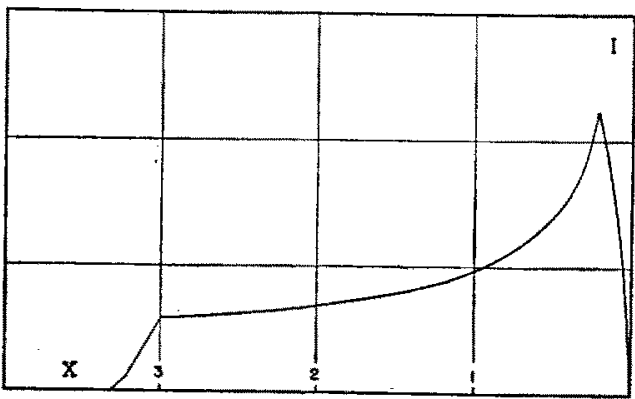

FIG. 4. The intensity distribution from a source of infinitesimal width. $X$ measured in $\mathrm{mm} ; p=120 \mathrm{~mm} ; D=37.8$ $\mathrm{mm} ; h=16 \mathrm{~mm}$.

where

$$
A=\frac{v}{\omega}(\pi-2 \theta) \sin \theta
$$

This equation may now be integrated with respect to $y_{0}$. The limits of this integration are in general from $-h / 2$ to $+h / 2$. However for small values of $A$ the limits must be modified so that the terms will not become imaginary.

Those particles for which $\alpha=\theta=0$ and $v=\omega p$ strike the $x y$ plane at $x-x_{0}=2(v / \omega)=2 p$ and also pass through the middle of the defining slit. If $\theta \neq 0$ there are two paths of the same radius for each value of $\theta$ which intersect the $x y$ plane at the same point, $x-x_{0}=2 p \cos \theta$. The defining slit stops all particles for which $\theta>\theta^{\prime}$, where $\theta^{\prime}$ is given approximately by

$$
D=2 p \sin \theta^{\prime} .
$$

When $\alpha \neq 0$ the cut-off by the defining slit becomes more complicated. In general the limits imposed by this slit apply to $\psi$ rather than to $\theta$, and some particles for which $\theta>\theta^{\prime}$ may still pass through the defining slit. The exact expression has been obtained but the algebra is rather lengthy. The results after two integrations are:

$$
\begin{array}{r}
I=\iint 2 n_{v} \sin \theta\left(A \frac{\pi-2}{2}\right) d \theta d x_{0} . \\
\text { from } \theta=0 \text { to } h=A \\
I=\iint 2 n_{v} \sin \theta\left[h \sin ^{-1} \frac{h}{A}\right. \\
\left.+\left(A^{2}-h^{2}\right)^{-}-A\right] d \theta d x_{0} \\
\text { from } h=A \text { to } \theta=\theta^{\prime} .
\end{array}
$$




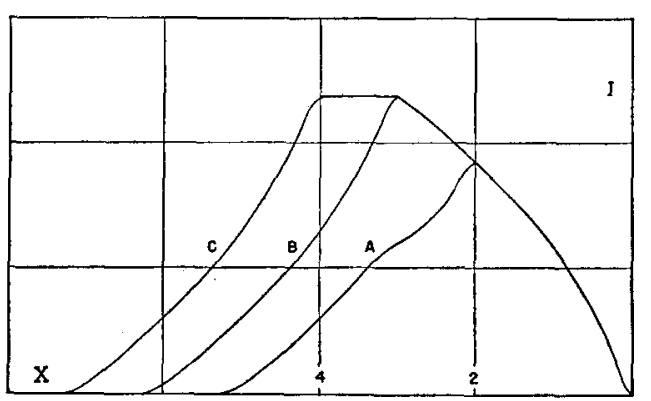

FIG. 5. The intensity distribution from a source of finite width. $X$ measured in $\mathrm{mm} ; p=120 \mathrm{~mm} ; D=37.8 \mathrm{~mm}$ $h=16 \mathrm{~mm} . A, B$, and $C$, represent cases where $l_{0}=2,3$ and $4 \mathrm{~mm}$, respectively.

For $\theta \geq \theta^{\prime}$ the intensity expression is practically equivalent to two straight lines as shown in Fig. 4. $\theta$ may be eliminated from these equations with Eq. (7). The final equations would give the intensity of particles of velocity $v=\omega p$, leaving a narrow vertical source of height $h$ and width $d x_{0}$ and entering a narrow vertical exit slit of height $h$ and width $d x$, as a function of the position of the source and slit. Fig. 4 represents the variation in intensity with $x$ as $x_{0}$ is held constant. The abscissa, $X$, is the distance from the point of maximum displacement and is defined by

$$
X=2 p-x+x_{0} \text {. }
$$

The intensity distribution for a source of finite width may be obtained by integrating Eqs. (13) and (14) and the corresponding equation for the other region with respect to $x_{0}$ between the proper limits. This integration is rather difficult unless further approximations are made. Since the integrand is a function of $x-x_{0}$ only, the integration in $x_{0}$ is equivalent to an integration in $x$ or $X$. The latter can be done easily by integrating the curve in Fig. 4 between the limits $X-l_{0}$ and $X$. The curve obtained in this fashion (Fig. 5) represents the intensity distribution in the plane of the exit slit due to a source of finite width. The abscissa again represents the distance from the point of maximum displacement from the source and is now defined by

$$
X=2 p-x+l_{0} / 2 .
$$

The shape of this distribution curve depends on the relative magnitudes of the width of the line due to imperfect focusing and that due to the width of the source. The latter width is precisely equal to the source width while the former depends on the limitation of the angle $\theta$ by the defining slit. In Fig. 5 are shown three typical curves. Curves $A, B$, and $C$ represent the cases of $l_{0}<,=,>$ the width due to the defining slit. It is apparent that the optimum condition occurs when the two are equal. Eqs. (7) and (15) combine to give

$$
2 p\left(1-\cos \theta^{\prime}\right)=X_{\max }=l_{0} .
$$

$\theta^{\prime}$ may be eliminated between Eqs. (12) and (17) :

$$
D=\left(4 p l_{0}-l_{0}^{2}\right)^{\frac{1}{2}} .
$$

This gives the optimum aperture, $D$, for a given source width, $l_{0}$.

A similar criterion might be used in connection with the curve of Fig. 4 in order to determine the best ratio of the width of the source to the height of the source and exit slit. The width of the line due to the limitation of $\alpha$ by the exit slit is equal to the value of $X$ at the peak of the curve in Fig. 4 . If the curve were terminated by the defining slit at twice this value of $X$, a sharper line than the one shown would be obtained. This would result in a greatly reduced intensity unless all dimensions were correspondingly increased. In practice it is not convenient to do this, because it would necessitate a larger pole gap. This would result in a less uniform field. The approximations used in the present derivation of the line shape would not be valid, but it would not be difficult to extend the method to a higher degree of approximation.

Curve $B$, Fig. 5 , shows the distribution in the plane of the exit slit, or the line shape, of particles with a single velocity, $v=\omega p$. It has been shown that the edge of the line, $X=0$, lies at a distance $2 p=2 v / \omega$ from the inside edge of the source, $x=l_{0} / 2$. For values of $v$ only slightly different from $\omega p$ the distribution curve will be approximately the same shape but will be displaced a distance equal to $2 \Delta v / \omega$. This is an approximation because the cut-off limits of the defining slit actually are functions of $v$. However they are not very sensitive to small changes in $v$ in the vicinity of $\omega p$. The transmission factor as a function of the path radius may be obtained by computing graphically for each velocity the area of the distribution curve $B$ (Fig. 5). This is precisely the same scheme by which curve $B$ was 


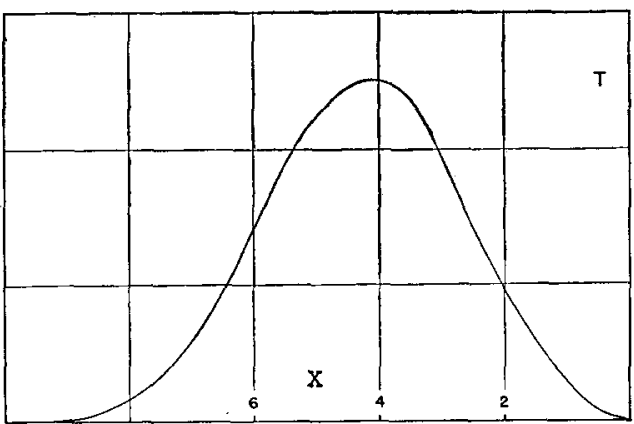

FIG. 6. The transmission factor of the slit system. $X$ measured in $\mathrm{mm} ; p=120 \mathrm{~mm} ; D=37.8 \mathrm{~mm} ; h=16 \mathrm{~mm}$; $l_{0}=l=3 \mathrm{~mm}$.

constructed and is equivalent to integrating curve $B$ between the limits $X-l$ and $X$. The shape of the resulting curve will depend on the width of the exit slit. Fig. 6 shows such a curve for the case $l_{0}=l$. The abscissa $X$ is defined by

$$
2 v / \omega=X+2 p-l \text {. }
$$

This curve may be interpreted in two different ways. It represents the relative transmission factors of the slit system for various velocities, or it represents the transmission factor for a single velocity as the magnetic field is varied. Therefore it is the line shape of particles of a single velocity. The best criterion for the resolving power of the spectrometer is the width, w, of this line at half the maximum intensity. It is important to obtain as high an intensity of particles entering the exit slit as is compatible with good resolution. Therefore it is desirable to find that ratio of source width to exit slit width which gives maximum intensity for a given resolution. The total intensity of particles of all velocities entering the exit slit is approximately proportional to the product of the widths of the source, defining slit and exit slit.

$$
I \sim l_{0} \cdot l \cdot D
$$

From Eq. (18) $D \sim l_{0}^{\frac{1}{2}}$ approximately. Hence,

Let

$$
I \sim l_{0}^{\frac{3}{2}} \cdot l \text {. }
$$

$$
\frac{l_{0}}{l}=C \text {. }
$$

For a given value of $C$ the ratio of the exit slit width to the half-value width may be shown to be very nearly a constant.

$$
\frac{l}{w}=B=f(C),
$$

hence

$$
I \sim C^{3 / 2} B^{5 / 2} w^{5 / 2} .
$$

There remains the determination of the value of $C$ which gives a maximum $I$ for a constant $w . B$ is a function of $C$ which is rather difficult to calculate, so it was determined graphically. The optimum condition was found to exist when the source and exit slit were of equal width.

If the source emits particles having a continuous spectrum of velocities the total number of particles leaving the exit slit is given by

$$
N=\int n_{\rho} T(\rho) d \rho
$$

where $n_{\rho}$ is the number of particles between $\rho$ and $\rho+d \rho$ in the true spectrum, and $T(\rho)$ is the relative transmission factor of the spectrometer for various radii of curvature, $\rho=v / \omega . T(\rho)$ is a function of the size and position of the slits and has been determined (Fig. 6) for the arrangement used in the present instrument. $n_{\rho}$ may be expanded around an average $\rho_{0}$ :

$$
n_{\rho}=n_{\rho_{0}}+\lambda\left(\rho-\rho_{0}\right)+\frac{\mu}{2}\left(\rho-\rho_{0}\right)^{2}+\cdots,
$$

where $\lambda$ is the slope and $\mu$ the curvature of the true spectrum at the point $\rho=\rho_{0}$. Therefore,

$$
\begin{aligned}
N=n_{\rho 0} \int T(\rho) d \rho+ & \lambda \int\left(\rho-\rho_{0}\right) T(\rho) d \rho \\
& +\frac{\mu}{2} \int\left(\rho-\rho_{0}\right)^{2} T(\rho) d \rho+\cdots .
\end{aligned}
$$

If $\int T(\rho) d \rho$ is normalized to unity a number proportional to the true number of particles, $N \rho_{0}$, may be obtained.

$$
\begin{aligned}
N_{\rho_{0}}=N-\lambda \int\left(\rho-\rho_{0}\right) T(\rho) d \rho \\
\\
\quad-\frac{\mu}{2} \int\left(\rho-\rho_{0}\right)^{2} T(\rho) d \rho+\cdots .
\end{aligned}
$$


$\rho_{0}$ may be chosen in such a way that

$$
\int\left(\rho-\rho_{0}\right) T(\rho) d_{\rho}=0 .
$$

Then,

$$
N_{\rho_{0}}=N-\frac{\mu}{2} \int\left(\rho-\rho_{0}\right)^{2} T(\rho) d \rho+\cdots
$$

Therefore if $\mu$ is small enough to make the correction term in Eq. (30) negligible, the observed number of particles is proportional to the true number of particles between $\rho_{0}$ and $\rho_{0}+\Delta \rho_{0}$. Eq. (29) was solved graphically for the case $l=l_{0}=3$ $\mathrm{mm}$ and $p=120 \mathrm{~mm}$. Under these conditions,

$$
\rho_{0}=p+0.632 \mathrm{~mm} \text {. }
$$

Eq. (31) holds for values of $p$ in the vicinity of $120 \mathrm{~mm}$.

The correction term in Eq. (30) may now be evaluated by the same method. Eq. (30) becomes

$$
N_{\rho_{0}}=N-0.285 \frac{d^{2} n}{d \rho^{2}}=N-0.285 \frac{H^{2}}{\rho_{0}^{2}} \frac{d^{2} n}{d H^{2}} .
$$

Since the correction is in general quite small the curvature of the observed spectrum may be used in place of that of the true spectrum. Eq. (32) then becomes

$$
N_{\rho_{0}}=N-0.285\left(H^{2} / \rho_{0}{ }^{2}\right)\left(d^{2} N / d H^{2}\right) .
$$

The order of magnitude of the maximum correction given by Eq. (33) has been found to be about 1 part in 5000 for most spectra.

This method of eliminating the error introduced in each measurement by the slope of the spectrum breaks down when the radius of curvature of the particles of maximum momentum falls within the limits selected by the spectrometer. Particles will continue to leave the exit slit until $H$ is increased beyond the value at which the maximum path radius is one-half the minimum distance between source and slit. Thus if $H \rho_{0}$ is used to determine the momentum there will be a "tail" on the spectrum extending a distance $H \rho_{0}-H(p-l / 2)$ beyond the true upper limit $H(p-l / 2)$. This amounts to about 1.8 percent for the slit system used at present. It is difficult to estimate exactly where the intensity becomes zero because the tail approaches zero yery gradually. It is possible to determine the shape of the tail from the transmission factor, provided the true shape of the spectrum is known in that region. Likewise the true shape may be obtained from the experimental curve by successive approximations. In practice the first or second approximation is sufficient. The experimental upper limit depends to some extent on the intensity of the source. The observed end point will correspond to that point of the true spectrum with its tail, which has the minimum observable intensity. If the source and slits are made larger the length of the tail is increased. However the intensity of the beam relative to the background is increased by a much larger factor (Eq. (21)). If the intensity is extremely low, the observed end point is far removed from the true end point. Under this condition the accuracy in determining the end point could be increased by decreasing the resolving power to gain more intensity. For a given available source intensity there will be an optimum size of slit system for the determination of the upper limit. An exit slit width of about $3 \mathrm{~mm}$ has been found to be satisfactory for most sources.

If the source emits gamma-rays which are internally converted, there will appear homogeneous groups of electrons. As the magnetic field is varied the intensity of electrons of these groups is given by the transmission factor curve (Fig. 6). The momentum of these electrons may be determined from the value of $H$ at any point of the measured curve and the value of $\rho$ from the corresponding point of the computed curve. If the point of maximum intensity is selected as the point of correspondence, the value of $\rho$ to use with the measured value of $H$ is :

$$
\rho=p+0.52 \mathrm{~mm}
$$

for the case of a 3-mm exit slit. The half-value width of the line is $3.85 \mathrm{~mm}$ or 1.6 percent. The resolution could be increased by decreasing the width of the slits and source. The ultimate resolution is limited only by the source intensity available and the accuracy with which the magnetic field can be maintained constant and measured.

When the magnetic field is changed the spectrometer selects particles from a different momentum region. Since the geometrical arrangement of the slit system is unchanged during the 
measurement of a spectrum, the particles are selected from equal intervals of the radius of curvature of their paths. In order to express the relative numbers of particles in terms of equal intervals of some other related parameter, $W$, it is necessary merely to divide each measurement by $d W / d \rho$. The momentum of a particle is proportional to $H \rho$, so to convert each measurement to extend over an equal momentum interval it is necessary to divide it by the magnetic field.

\section{Corrections to the Counting Rate}

The process of measuring a spectrum with the variable field spectrometer consists essentially in recording the counting rate of the GeigerMüller counter for various settings of the magnetic field. These measurements do not represent the true distribution of particles, since there are several other factors which influence the counting rate. The effect of the resolving power of the instrument has already been discussed. It was shown that this could be practically eliminated by the proper choice of the average radius of curvature of the paths. The corrections to be considered now are those which apply to the counting rate. The counting rate is influenced by the five following factors: (1) The magnitude of the counting rate; (2) The background of the counter; (3) The decay of the source; (4) The efficiency of the counter; (5) The absorption and scattering of the particles by the window of the counter.

Lifschutz and Duffendack ${ }^{10}$ have experimentally verified the counting loss theory of Ruark and Brammer. They have shown that the formula

$$
n=n_{0} /\left(1+n_{0} \sigma\right)
$$

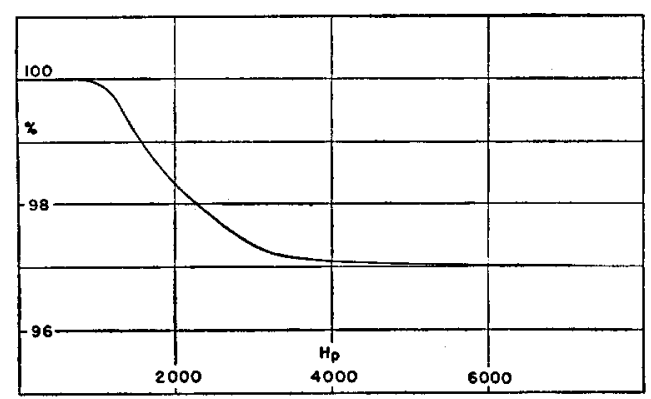

FIG. 7. Variation of the efficiency of the Geiger-Müller counter. where $n$ is the observed counting rate, $n_{0}$ the true counting rate, and $\sigma$ the resolution time of the Geiger-Müller counter, is valid for the recording circuit previously described. $\sigma$ may be determined from the maximum counting rate when a very strong source is brought near the counter:

$$
\sigma=1 / \eta_{\max } \text {. }
$$

For the counter used in the spectrometer $\sigma=4.95 \times 10^{-6} \mathrm{~min}$. The losses computed in this fashion were checked by an addition test at a counting rate of 10,000 per minute. For counting rates up to 1000 per minute this correction amounts to less than 0.5 percent, which may usually be neglected.

The background of the counter is determined when the beam is cut off near the exit slit by a Bakelite gate, $G$. The gate is designed so that some, but not all, of the particles scattered from the interior of the tank are recorded as background. However, the scattering is so low that no change in background has been observed when the magnetic field is varied. The background has been fairly constant from day to day. Its average value is about 4.5 counts per minute when the counter is in place between the pole tips, and about 16 counts per minute when the counter is out in the room. The iron of the magnet fortunately reduces the cosmic-ray intensity considerably. The background should be subtracted from the counting rate after the latter is corrected for the counting rate losses, but before the other corrections are applied. The remaining three corrections are all multiplicative, hence may be applied in any order.

The decay of the source may be determined by repeating measurements at various times. If the spectrum is composite, approximate separation of its components can be made by decay measurements at differentfield settings. If gamma-rays are present their half-lives may be determined separately.

If the Geiger-Müller counter were equally efficient for particles of all energies, the absolute value of efficiency would make no difference in the shape of the spectrum. Only the dependence of the efficiency on the energy of the particles need be considered. It can be easily shown that the probability of forming at least one ion pair 


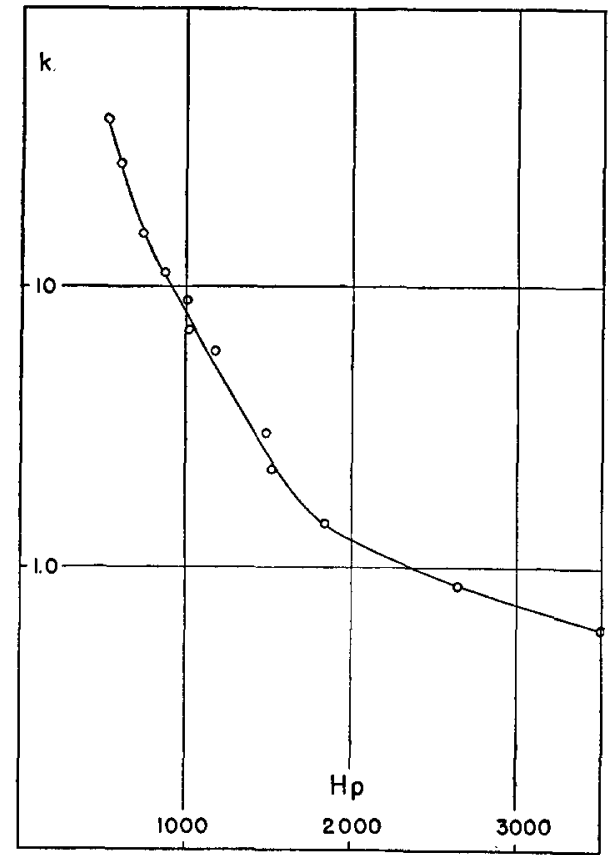

FIG. 8. Variation of the absorption coefficient of the window.

in the counting volume of length $L$ is

$$
\text { Probability }=1-e^{-L / q},
$$

where $q$ is the mean free path of the primary particle in the gas of the counter. This will represent the efficiency of the counter if there is a high probability that an ion so formed will produce a discharge. This can be realized by applying a high voltage to the counter. $q$ has been measured in hydrogen for various momenta by Williams and Terroux. ${ }^{13}$ By varying the gas pressure in the counter a good estimation of the efficiency can be made, from which $L$ may be computed. For the counter previously described, operating at 2000 volts and with hydrogen at a pressure of $25 \mathrm{~cm}$ of mercury, $L$ was thus indirectly measured to be $18 \mathrm{~mm}$ which is the physical length of the cathode. From the

${ }^{13}$ Williams and Terroux, Proc. Roy. Soc. A126, 289 (1930). experimental values of $L$ and $q$ the dependence of the counting efficiency upon the momentum of the incoming beta-particles can be computed (Fig. 7) from Eq. (37).

The final correction to the counting rate has to do with the scattering and absorption of the particles by the counter window. This correction becomes very important at the lower energies, and the accuracy with which it can be made determines the lower limit of energy which can be measured. Absorption curves were taken at different energies by interposing different thicknesses of the window material in front of the exit slit. The absorption obeyed an exponential law quite well for all but the extremely low energies. The absorption coefficients were calculated and plotted against the momentum (Fig. 8 ). The window correction is given by the equation

$$
N_{0}=N e^{k t / 100},
$$

where $N_{0}$ is the true counting rate, $N$ the observed counting rate, $t$ the window thickness, and $k$ the absorption coefficient taken from the experimental curve. The unit of thickness is microns $/ \eta$, where $\eta$ is the index of refraction of the window material. The absorption is small and nearly constant for particles of momentum $>1800$ $\mathrm{H} \rho$. Between 1800 and $700 \mathrm{H} \rho$ the absorption coefficient increases very rapidly. In this region large angle scattering is probably the most important factor in reducing the intensity of the beam. Below $700 \mathrm{H \rho}$ the absorption coefficient increases still more rapidly, because the window begins to absorb the beam strongly. Because of the scattering at low energies, these absorption coefficients are not a property of the window alone, but depend also on the geometry of the counter and exit slit.

The authors are indebted to Professors J. M. Cork and S. A. Goudsmit and Dr. H. R. Crane for many helpful discussions. This work has been made possible by the Horace $H$. Rackham trust fund. 